Swarthmore College

Works

Physics \& Astronomy Faculty Works

Physics \& Astronomy

3-1-2003

\title{
X-Ray Emission Line Profile Modeling Of Hot Stars
}

Roban Hultman Kramer , '03

Stephanie Korinne Tonnesen , '03

David H. Cohen

Swarthmore College, dcohen1@swarthmore.edu

S. P. Owocki

A. Ud-Doula

See next page for additional authors

Follow this and additional works at: https://works.swarthmore.edu/fac-physics

Part of the Astrophysics and Astronomy Commons

Let us know how access to these works benefits you

\section{Recommended Citation}

Roban Hultman Kramer , '03; Stephanie Korinne Tonnesen , '03; David H. Cohen; S. P. Owocki; A. Ud-Doula; and J. J. MacFarlane. (2003). "X-Ray Emission Line Profile Modeling Of Hot Stars". Review Of Scientific Instruments. Volume 74, Issue 3. 1966-1968. DOI: 10.1063/1.1535240

https://works.swarthmore.edu/fac-physics/56

This work is brought to you for free by Swarthmore College Libraries' Works. It has been accepted for inclusion in Physics \& Astronomy Faculty Works by an authorized administrator of Works. For more information, please contact myworks@swarthmore.edu. 


\section{Authors}

Roban Hultman Kramer , '03; Stephanie Korinne Tonnesen , '03; David H. Cohen; S. P. Owocki; A. Ud-Doula; and J. J. MacFarlane 


\section{A|P| $\mid \begin{aligned} & \text { Review of } \\ & \text { Scientific Instruments }\end{aligned}$}

\section{X-ray emission line profile modeling of hot stars}

Roban H. Kramer, Stephanie K. Tonnesen, David H. Cohen, Stanley P. Owocki, Asif ud-Doula, and Joseph J. MacFarlane

Citation: Review of Scientific Instruments 74, 1966 (2003); doi: 10.1063/1.1535240

View online: http://dx.doi.org/10.1063/1.1535240

View Table of Contents: http://scitation.aip.org/content/aip/journal/rsi/74/3?ver=pdfcov

Published by the AIP Publishing

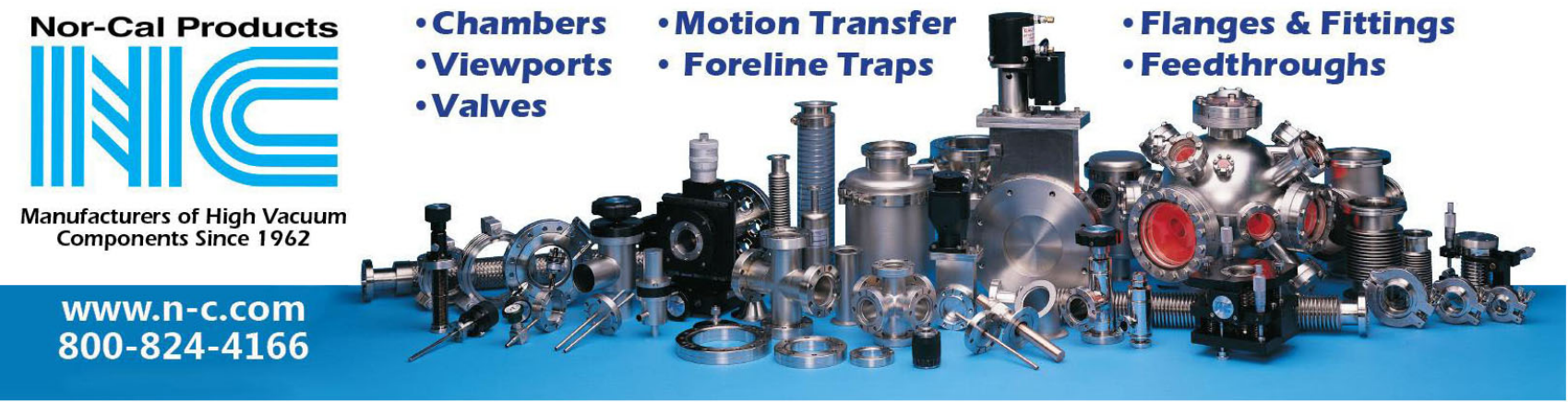




\title{
X-ray emission line profile modeling of hot stars
}

\author{
Roban H. Kramer \\ Swarthmore College, Swarthmore, Pennsylvania 19081 and Prism Computational Sciences, Madison, \\ Wisconsin 53711
}

Stephanie K. Tonnesen

Swarthmore College, Swarthmore, Pennsylvania 19081

David H. Cohen

Swarthmore College, Swarthmore, Pennsylvania 19081 and Prism Computational Sciences, Madison, Wisconsin 53711

Stanley P. Owocki and Asif ud-Doula

Bartol Research Institute, University of Delaware, Newark, Delaware 19716

Joseph J. MacFarlane

Prism Computational Sciences, Madison, Wisconsin 53711

(Presented on 10 July 2002)

\begin{abstract}
The launch of high-spectral-resolution x-ray telescopes (Chandra, XMM) has provided a host of new spectral line diagnostics for the astrophysics community. In this paper we discuss Doppler-broadened emission line profiles from highly supersonic outflows of massive stars. These outflows, or winds, are driven by radiation pressure and carry a tremendous amount of kinetic energy, which can be converted to $\mathrm{x}$ rays by shock-heating even a small fraction of the wind plasma. The unshocked, cold wind is a source of continuum opacity to the $\mathrm{x}$ rays generated in the shock-heated portion of the wind. Thus the emergent line profiles are affected by transport through a two-component, moving, optically thick medium. While complicated, the interactions among these physical effects can provide quantitative information about the spatial distribution and velocity of the x-ray-emitting and absorbing plasma in stellar winds. We present quantitative models of both a spherically symmetric wind and a wind with hot plasma confined in an equatorial disk by a dipole magnetic field. (C) 2003 American Institute of Physics. [DOI: 10.1063/1.1535240]
\end{abstract}

\section{INTRODUCTION}

Ultraviolet spectra of $\mathrm{O}$ and $\mathrm{B}$ stars (with luminosities up to $L=10^{6} \quad \mathrm{~L}_{\odot}$ and surface temperatures $T \geqslant 3 \mathrm{~T}_{\odot}$ ) show the signatures of rapidly expanding winds, with velocities on the order of a few $1000 \mathrm{~km} \mathrm{~s}^{-1}$, densities of order $10^{10} \mathrm{~cm}^{-3}$, and mass-loss rates up to $10^{-5} \mathrm{M}_{\odot} \mathrm{yr}^{-1}$. These stars are detected to have soft-x-ray luminosities of $10^{-7}$ times their total (bolometric) luminosities. ${ }^{1}$ In cooler stars, like the Sun, $x$ rays are produced in coronas, which are high-temperature regions near the surface of the star, and their x-ray line widths are primarily indicative of thermal broadening. The line widths observed in x-ray spectra of hot stars, however, provide evidence that the emission originates in extended, high-velocity winds. ${ }^{2}$

The Chandra X-Ray Observatory has the spectral resolution to resolve Doppler-broadened lines from hot stars, providing quantitative information about the geometry and kinematics of the wind and putting important constraints on physical models that may explain the observed $x$ rays. Chandra uses a set of concentric parabolic and hyperbolic mirrors with an effective area of about $10 \mathrm{~cm}^{2}$ to focus $\mathrm{x}$ rays on its instrument package. The Medium Energy Grating (MEG) provides a resolution of $\Delta \lambda=0.023 \AA$ from 0.4 to $5.0 \mathrm{keV}$ (31 to $2.5 \AA$ ).

\section{SPHERICALLY SYMMETRIC WIND MODELS}

An optically thin, spherically expanding, x-ray-emitting plasma produces symmetric line profiles centered on the rest wavelengths of the lines with a shape determined entirely by the velocity and emissivity structure of the plasma. Two effects can change the symmetry of the line. The star itself occults part of the plasma, while cold components of the wind absorb $\mathrm{x}$ rays, meaning photons traveling along longer lines of sight or through more dense wind regions will be less likely to reach the observer. In a radially expanding wind, the observer sees blueshifted emission from the approaching material on the near side of the wind and redshifted emission from the receding material on the far side. Thus an optically thick wind has the effect of removing photons from the red side of the profile, shifting the line center blueward and altering the shape of the line.

Owocki and Cohen $^{3}$ have numerically integrated a spherically-symmetric wind model to determine the shape of line profiles produced in a wind characterized by four parameters. The wind velocity at a distance $r$ from the center of the star is assumed to be of the form $v(r)=v_{\infty}\left(1-R_{*} / r\right)^{\beta}$ (a " $\beta$ velocity law"), where $R_{*}$ is the stellar radius, $v_{\infty}$ is the terminal velocity of the wind (determined from ultraviolet spectra), and $\beta$ is a free parameter of order unity. Given a constant mass-loss rate, the density at any point in a 

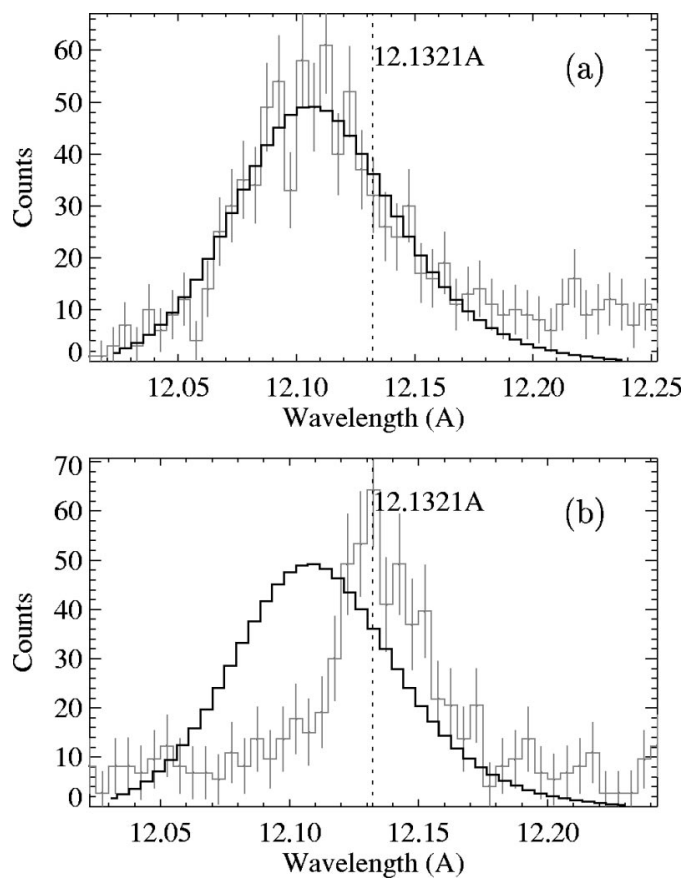

FIG. 1. Chandra MEG ( +1 and -1 order summed) spectra (with error bars) of the Ne X Lyman $\alpha$ line in (a) $\zeta$ Puppis, and (b) $\theta^{1}$ Orionis C, with the best-fit spherically-symmetric wind model for $\zeta$ Puppis (in black). The parameters for the best-fit model are $\beta=1, q=1.2, R_{0}=1.1 R_{*}$, and $\tau_{*}$ $=2.5$. The area under the model curve is normalized to the area under the data curve.

smoothly expanding, spherically symmetric wind can be determined from the velocity law. The emissivity of the wind is taken to be zero below some radius $R_{0}$, and to fall off like $n^{2} r^{-q}$ above that, where $n$ is the density of the wind and $q$ is a free parameter. The optical depth of the wind is characterized by the parameter $\tau_{*}$, which is defined so that, in a constant velocity wind $\left(\beta=0, v=v_{\infty}\right), \tau_{*}$ is the radial optical depth at the surface of the star, and the radius of optical depth unity is $\left.r\right|_{\tau=1}=\tau_{*} R_{*}$. The parameters $\beta, q, R_{0}$, and $\tau_{*}$ offer enough flexibility to characterize a variety of physical models, including coronal models (by setting $q$ and $\beta$ to high values and $R_{0}=R_{*}$ ).

All lines produced in an extended wind of this form will be skewed to the blue side of line center by the removal of photons from the red edge of the profile. Chandra observations of the star $\zeta$ Puppis $^{4}$ reveal broad lines with a significant blueward skew, suggesting an extended, spherical wind model may be able to explain the shape of the profiles.

Preliminary work on fitting the Owocki and Cohen model to lines in $\zeta$ Puppis suggest that good fits may be obtained with physically reasonable parameters. Figure 1(a) shows the Chandra MEG (sum of +1 , and -1 order) spectrum of the $12.13 \AA$ Ne X Lyman $\alpha$ line of $\zeta$ Puppis along with the best-fit line profile.

Other hot stars, however, show much more symmetric profiles. Figure 1(b) shows the same Ne X line of $\theta^{1}$ Orionis $\mathrm{C}$ along with the model that fit the line in $\zeta$ Puppis. No spherically symmetric wind model that includes absorption can fit the relatively narrow and symmetric line from this star.
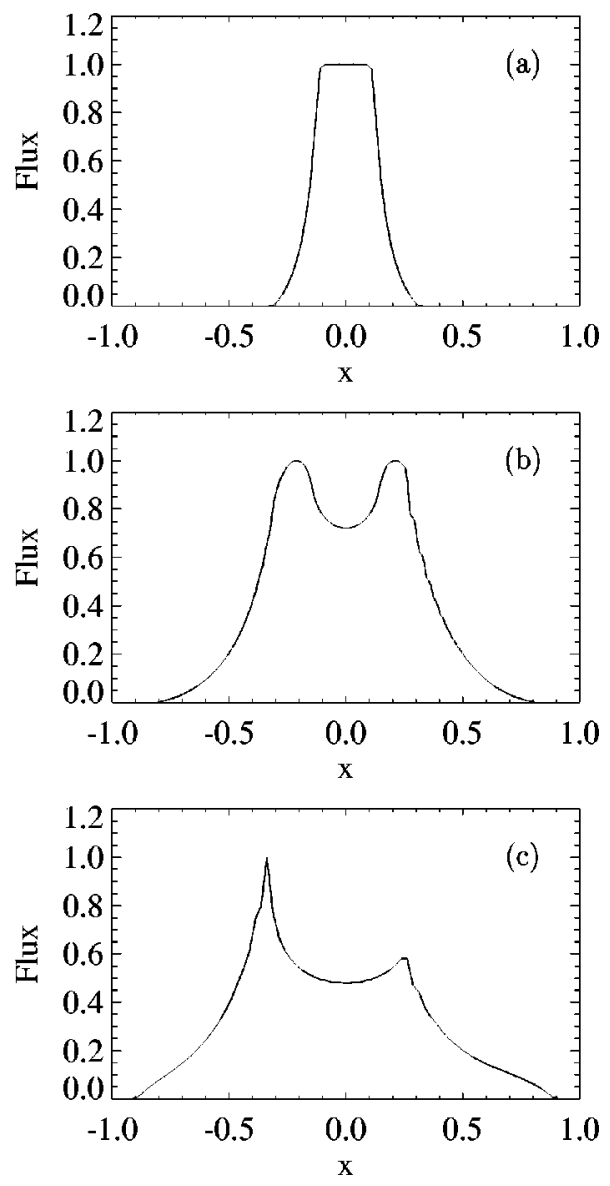

FIG. 2. Line profiles of an equatorial disk for an observer at three different viewing angles (a) $0^{\circ}$ (magnetic-pole on), (b) $45^{\circ}$, and (c) $90^{\circ}$ (along magnetic equator). The horizontal axis shows the wavelength scaled to the terminal velocity of the wind, $x=v / v_{\infty}=\left(\lambda-\lambda_{0}\right) c / \lambda_{0} v_{\infty}$. The flux is normalized to the maximum value. Note that the asymmetry in the profile is due to occultation of a portion of the redshifted part of the wind by the star.

\section{MODELING NONSPHERICALLY SYMMETRIC WINDS}

A promising alternative to spherically symmetric models is the Magnetically Confined Wind Shock (MCWS) model in which a strong $(\mathrm{kG})$, large-scale dipole magnetic field originates from the star's surface and directs the stellar outflow. This field directs the wind toward the magnetic equator, where flows from opposite hemispheres collide, causing strong shocks and heating the wind plasma to $T \approx 10^{7} \mathrm{~K}$. This theory, originally proposed by Babel and Montmerle, ${ }^{5}$ lately has gained currency due to the direct detection of an $1100 \mathrm{G}$ field on $\theta^{1}$ Orionis $\mathrm{C}$ (Ref. 6) and the detailed magnetohydrodynamic modeling by ud-Doula and Owocki. ${ }^{7}$

As an initial exploration of this model, we have numerically synthesized emission line profiles of equatorial x-rayemitting flows. In Fig. 2 we show the results of a simple model based on a radially directed flow confined to the magnetic equator of a hot star (using a $\beta$ velocity law, with the flow confined to within $20^{\circ}$ of the equator). Because stellar magnetic fields are often tilted with respect to the rotation axis, our viewing orientation changes with rotation phase. In the figure we show a simulated line profile from three differ- 
ent angles: $0^{\circ}$ (magnetic pole-on), $45^{\circ}$, and $90^{\circ}$ (equator-on). The characteristics of these profiles are due to (the changing) projected radial velocity of the x-ray-emitting wind, as well as (viewing-angle dependent) occultation by the star.

Clearly, the rotation phase can affect the line profiles, which is a conclusion we will be testing against recently obtained multiphase Chandra observations of $\theta^{1}$ Orionis $\mathrm{C}$ itself. This star has a $45^{\circ}$ angle between the magnetic and rotation axes, ${ }^{6}$ and a viewing angle that is also tilted by $45^{\circ}$ with respect to the rotation axis, giving us a full range of viewing angles with respect to the star's magnetic axis. Future modeling work will include the effects of absorption by the cold wind and also take into account more realistic and complex flow morphologies, including spectral post processing of numerical MHD simulations.

${ }^{1}$ T. Chlebowski, F. R. Harnden, and S. Sciortino, Astrophys. J. 341, 427 (1989).

${ }^{2}$ S. M. Kahn, M. A. Leutenegger, J. Cottam, G. Rauw, J.-M. Vreux, A. J. F. den Boggende, R. Mewe, and M. Güdel, Astron. Astrophys. 365, L312 (2001).

${ }^{3}$ S. P. Owocki and D. H. Cohen, Astrophys. J. 559, 1108 (2001).

${ }^{4}$ J. P. Cassinelli, N. A. Miller, W. L. Waldron, J. J. MacFarlane, and D. H. Cohen, Astrophys. J. Lett. 554, L55 (2001).

${ }^{5}$ J. Babel and T. Montmerle, Astron. Astrophys. 323, 121 (1997).

${ }^{6}$ J.-F. Donati, J. Babel, T. J. Harries, I. D. Howarth, P. Petit, and M. Semel, Mon. Not. R. Astron. Soc. 333, 55 (2002).

${ }^{7}$ A. ud-Doula and S. P. Owocki, Astrophys. J. 576, 413 (2002). 